\title{
Connecting flow structures and heat flux in turbulent Rayleigh-Bénard convection
}

\author{
Erwin P. van der Poel, Richard J. A. M. Stevens, and Detlef Lohse \\ Physics of Fluids Group, Department of Science and Technology, Mesa+ Institute, and J. M. Burgers center for Fluid Dynamics, \\ University of Twente, 7500 AE Enschede, The Netherlands
}

(Received 13 July 2011; published 19 October 2011)

\begin{abstract}
The aspect ratio $(\Gamma)$ dependence of the heat transfer (Nusselt number $\mathrm{Nu}$ in dimensionless form) in turbulent (two-dimensional) Rayleigh-Bénard convection is numerically studied in the regime $0.4 \leqslant \Gamma \leqslant 1.25$ for Rayleigh numbers $10^{7} \leqslant \mathrm{Ra} \leqslant \mathrm{Ra}^{9}$ and Prandtl numbers $\mathrm{Pr}=0.7$ (gas) and 4.3 (water). $\mathrm{Nu}(\Gamma$ ) shows a very rich structure with sudden jumps and sharp transitions. We connect these structures to the way the flow organizes itself in the sample and explain why the aspect ratio dependence of $\mathrm{Nu}$ is more pronounced for small Pr. Even for fixed $\Gamma$ different turbulent states (with different resulting $\mathrm{Nu}$ ) can exist, between which the flow can or cannot switch. In the latter case the heat transfer thus depends on the initial conditions.
\end{abstract}

DOI: 10.1103/PhysRevE.84.045303

PACS number(s): 47.27.te

The physicists' view on fully developed turbulence has been dominated by Kolmogorov's seminal work [1], postulating the universality of the small scales. Real-world flows, however, have walls and boundaries. How do these geometric properties affect the global transport characteristics of the flow such as heat or momentum transfer? The common view is that in the fully turbulent state, due to the large fluctuations of the system, the phase space is fully explored by the dynamics of the flow and that universality implies an, at most, weak dependence on the boundary conditions, clearly without any jumps in global transport properties.

Only recently the community became aware of the possibility of the coexistence of different turbulent states, with firstor second-order phase transitions in between them. Examples include different turbulent states in von Kármán flow ("French washing machine" [2]), in magnetohydrodynamic turbulence in a closed system (von Kármán turbulent liquid sodium experiment) [3], in turbulent rotating Rayleigh-Bénard (RB) flow [4], or in turbulent rotating spherical Couette flow [5], and possibly even in high Rayleigh number turbulent RB flow in a cylindrical cell of aspect ratio $\Gamma=1 / 2$ [5-7] and $\Gamma=0.23$ [8]. That transitions between different flow states occur in closed flows at a low degree of turbulence has been well known and explored in the context of spatial-temporal chaos and pattern formation (see, e.g., Ref. [9]), but that they also occur between turbulent states clearly came as a surprise.

In this Rapid Communication we will numerically show that different turbulent states with first- and second-order phase transitions in between them and even the coexistence of different turbulent states also exist in an even simpler geometry, namely, in a two-dimensional (2D) RB sample. The advantages of the 2D geometry are (i) that a flow visualization is much easier, (ii) that the complicated axial and torroidal dynamics of three-dimensional (3D) RB flow [10] does not complicate or even obscure the flow field analysis, and (iii) that it is numerically cheaper so that a good resolution in the aspect ratio $\Gamma$ (i.e., many different runs with different $\Gamma$ ) becomes feasible. These features will enable us to reveal the connection between the flow organization and the heat-transfer properties. However, we stress that these connections also exist in 3D RB flow, and working them out from experimental measurements had been pioneered by Xia and co-workers [11].
The main control parameter of this study will be the aspect ratio $\Gamma$, whose effect on the turbulent $\mathrm{RB}$ flow has not yet been extensively studied, to the best of our knowledge, with only a few theoretical and numerical studies [12-14]. The main reason for this shortcoming is that it is experimentally very difficult to vary $\Gamma$ in $3 \mathrm{D}$ cylindrical samples, as each $\Gamma$ requires a new sample. That is why in experiment the $\Gamma$ resolution has hitherto been insufficient to detect transitions between different turbulent states. In their review Ahlers et al. [10] conclude, based on a small number of experiments with few different $\Gamma$ [15-17], that the "weak $\Gamma$-dependence [of the heat transfer] suggests an insensitivity to the nature of the large-scale convection." We will show that in 2D RB this is definitely not the case.

One may argue that the flow phenomena in 3D are different and richer, which is correct. However, various 2D RB flow simulations [18-25] have revealed the value of this approach for a better understanding of turbulent RB convection and the analogies to 3D RB flow. In Ref. [24] we could even reveal a one-to-one analogy between the flow organization in our 2D numerical simulations and in experiments in a quasi-2D sample of the same aspect ratio $\Gamma=1$.

The code on which the results in this Rapid Communication are based is a fourth-order finite-difference discretization of the incompressible Oberbeck-Boussinesq equations. It has been described and tested in detail in Ref. [22]. The velocity boundary conditions on the walls are no slip. The (relative) temperature is fixed at $\Delta / 2$ at the bottom plate and $-\Delta / 2$ at the top plate, with adiabatic side-wall conditions. The grid resolution obeys the strict criteria formulated in Ref. [26]. The imposed initial condition is a single-roll state with a linear temperature profile. The sample aspect ratio is $\Gamma=D / H$, where $D$ is the sample width and $H$ the sample height. Times are given in multiples of large eddy turnover times $t_{E}:=4 \pi /\left\langle\left|\omega_{c}(t)\right|\right\rangle$, where $\omega_{c}$ is the center vorticity. Next to $\Gamma$, the other control parameters are the Rayleigh number Ra (the dimensionless temperature difference between top and bottom) and the Prandtl number $\operatorname{Pr}=v / \kappa$, where $v$ and $\kappa$ are kinematic viscosity and thermal diffusivity, respectively. The system responds with the heat transfer from bottom to top (in dimensionless form: Nusselt number $\mathrm{Nu}$ ) and the turbulence intensity (in dimensionless form: Reynolds number $\mathrm{Re}$ ). 

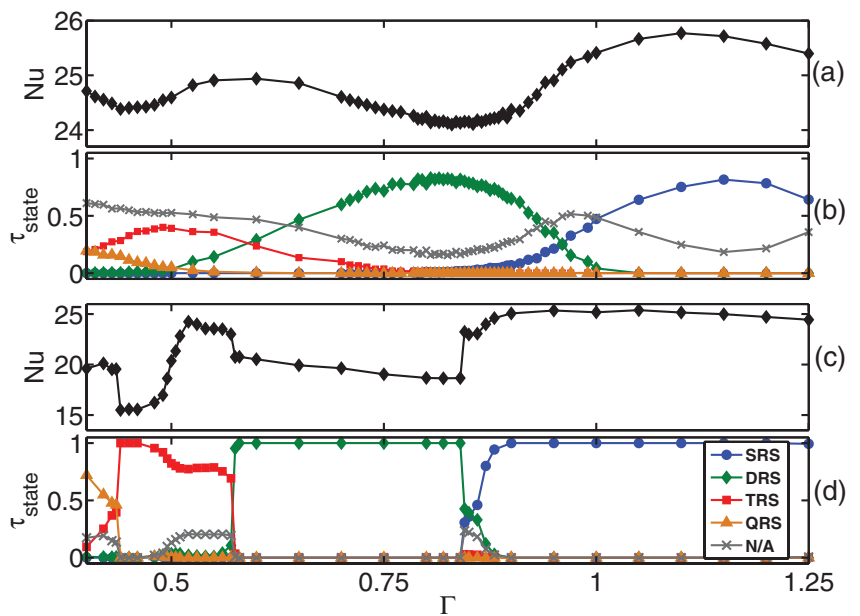

FIG. 1. (Color online) $\mathrm{Nu}(\Gamma)$ for $\mathrm{Ra}=10^{8}$ and (a) $\mathrm{Pr}=4.3$ and (c) $\operatorname{Pr}=0.7$ (averaging time $>10^{3} t_{E}$ ). The accompanying figures (b) and (d) show the respective relative time the system is in the singleroll state (SRS), double-roll state (DRS), triple-roll state (TRS), and quadruple-roll state (QRS). Additionally, the extent of time that no roll state could be identified is indicated.

In Fig. 1 we show $\mathrm{Nu}(\Gamma)$ for $\mathrm{Ra}=10^{8}$ and $\mathrm{Pr}=0.7$ and 4.3 , resulting from a very large averaging time of more than $10^{3} t_{E}$, for which we have checked the statistical convergence. For the lower $\mathrm{Pr}$ the curve shows variations in $\mathrm{Nu}$ of up to $35 \%$, with abrupt jumps and sharp transitions. From a visual inspection of flow movies we deduce that these jumps are associated with transitions in the overall flow organization from one to two vertically stacked rolls (at $\Gamma \approx 0.9$ ) and from two to three rolls (at $\Gamma \approx 0.55$ ). We automize this flow analysis by measuring the zeros in the vorticity along the center vertical axis, i.e., the zeros in $\omega(x=D / 2, z)$. A state is not counted if its lifetime is shorter than $t_{E}$. The results for the relative time in a certain state is shown in Fig. 1 . For $\operatorname{Pr}=0.7$ this analysis confirms that the transitions are rather sharp, with the coexistence of different roll states only in a small $\Gamma$ range. Next to the jumps, relatively sharp transitions in $\mathrm{Nu}(\Gamma)$ can be observed. As an example we discuss the $35 \%$ increase in $\mathrm{Nu}$ from $\Gamma=0.45$ to $\Gamma=0.55$, namely, from $\mathrm{Nu}=16$ to $\mathrm{Nu}=24$. This coincides with a developing instability of the triple-roll state (TRS): The sample becomes too wide for three rolls so that the three-roll structure breaks down from time to time. This breakdown shuffles warm fluid upward and cold fluid downward, leading to a larger temperature gradient across the thermal boundary layers (BLs) and thus to an increased heat flux. Typical snapshots of the temperature field in this regime are shown in Fig. 2; the movies can be seen in the Supplemental Material [27].

The enhanced vortex mobility at $\Gamma=0.55$ as compared to $\Gamma=0.45$ can also be deduced from the positions of the vortex centers, which we identify by a noniterative ellipse fit [28] coupled with a vortex criterium based on the nonzero imaginary part of the velocity gradient tensor's eigenvalues. The 2D probability density functions (PDFs) of these positions are visualized in Fig. 2(d) (for $\Gamma=0.45$ ) and Fig. 2(e) (for $\Gamma=0.55)$. While in the former case we see three pronounced sharp peaks, reflecting the stability of that flow configuration,

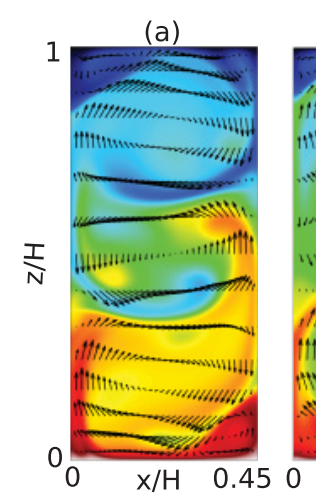

(b)
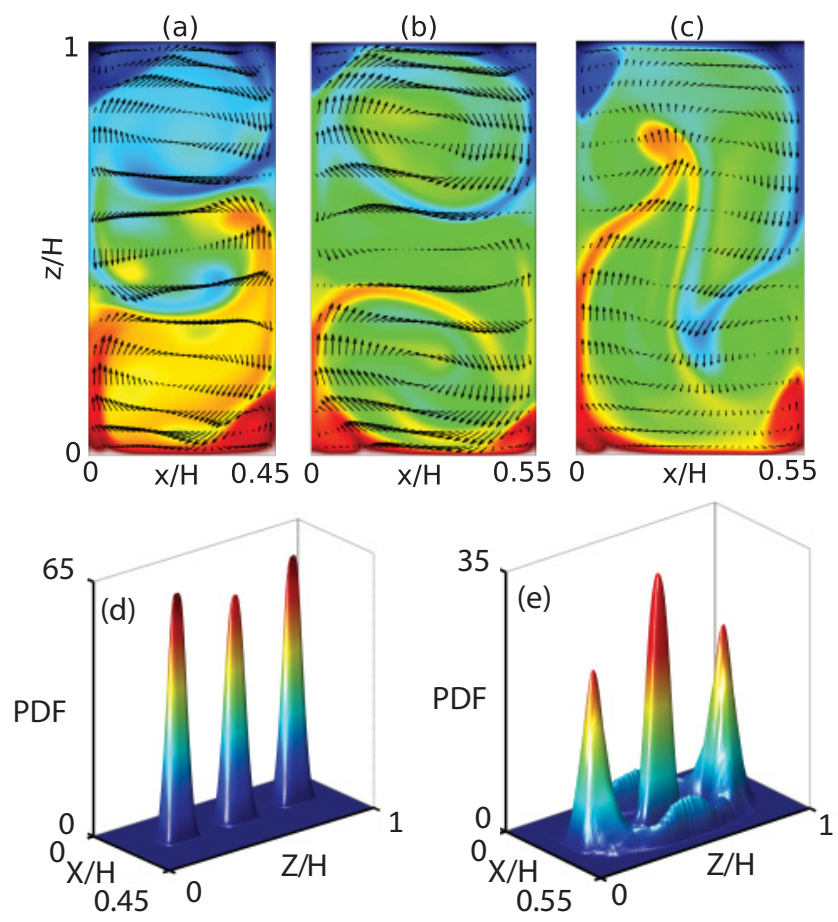

FIG. 2. (Color online) Snapshots of the temperature field for $\mathrm{Ra}=10^{8}$ and $\operatorname{Pr}=0.7$ for (a) $\Gamma=0.45$ with the flow in a very stable TRS and (b), (c) $\Gamma=0.55$ with the flow in an unstable TRS. The top and bottom rolls in (b) grow until collision (c), which causes enhanced mixing and heat transport. Corresponding videos can be seen as Supplementary Material [27]. The positions of the roll centers are automatically tracked and their PDFs as function of $(x, z)$ are shown in (d) for $\Gamma=0.45$ and (e) for $\Gamma=0.55$.

in the latter case the peaks are smeared out, showing the vortex mobility which contributes to the enhanced heat flux.

We now come to the case of large $\operatorname{Pr}=4.3$. Here the transitions in $\mathrm{Nu}(\Gamma)$ are much smoother as compared to the $\operatorname{Pr}=0.7$ case [compare Figs. 1(b) and 1(d)] with wide $\Gamma$ regimes of coexistence between different states, between which the dynamical evolution of the flow meanders, as, e.g., seen from temperature field snapshots of Fig. 3, the movie (a)

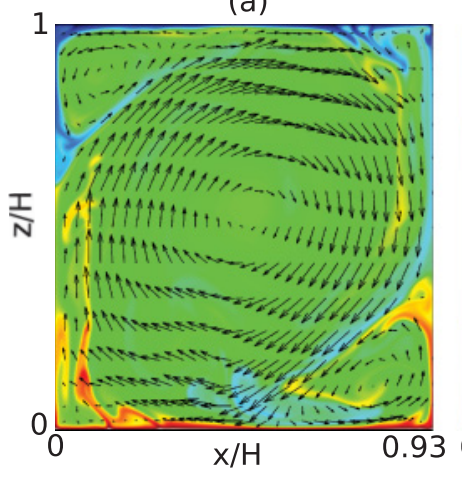

(b)

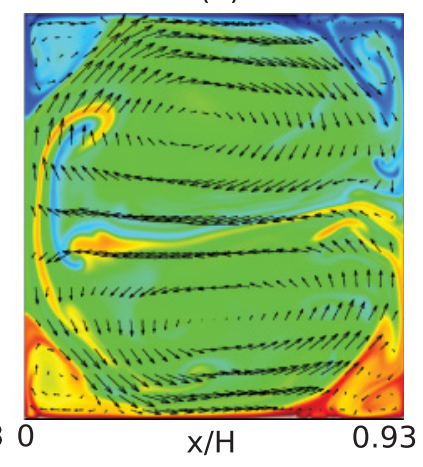

FIG. 3. (Color online) Two temperature snapshots for $\mathrm{Ra}=10^{9}$, $\operatorname{Pr}=4.3$, and $\Gamma=0.93$. The system is either in the SRS (a) or DRS (b). The corresponding movie is available online as Supplemental Material [27]. Heat transport efficiency differs between these states-see Fig. 4. 


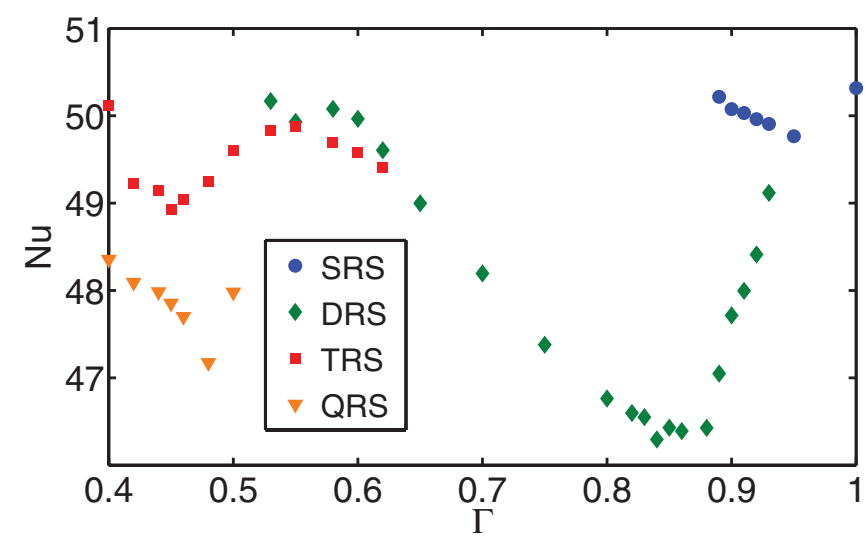

FIG. 4. (Color online) Conditionally averaged $\mathrm{Nu}(\Gamma)$ for the various states SRS, DRS, and TRS, which can be identified at $\mathrm{Ra}=10^{9}$ and $\operatorname{Pr}=4.3$. For fixed $\Gamma$ the flow jumps in between these different states.

in the Supplemental Material [27], and the time series of the Nusselt number. For these larger Pr the dynamics is much richer as the flow keeps on switching between the different states. The reason for this lies in the plume dynamics: Whereas for $\operatorname{Pr}=0.7$ the larger thermal diffusivity tends to smoothen out temperature differences between different flow parcels, for larger $\operatorname{Pr}=4.3$ the plumes keep their thermal energy for a longer time. Thus the plumes, which feed the competing rolls as described in Ref. [24], have a longer lifetime, which leads to more lively roll dynamics. Therefore a $100 \%$ dominance of a certain roll state, common for small Pr, does not occur.

Based on the state analysis, the heat flux can be conditionally averaged on the SRS, DRS, or TRS. The results for these conditional heat fluxes are shown in Fig. 4. For the vertically arranging vortices it again holds that the stable states with $n$ rolls enable larger heat transfer than the stable states with $n+1$ rolls. For $n=1$ and $n=2$ the difference is $\sim 5 \%$ for $\mathrm{Ra}=10^{9}$, $\operatorname{Pr}=4.3$. Remarkably, that value is similar to what one would expect for the 3D situation, based on an extrapolation toward $\mathrm{Ra}=10^{9}$ of the $\mathrm{Nu}$ measurements for the SRS and DRS in Ref. [29] (for $4 \times 10^{9} \leqslant \mathrm{Ra} \leqslant 10^{11}$ ), which roughly gives $3 \%$.

We also found a case for which the final state and thus $\mathrm{Nu}$ depend on the initial conditions of the flow field (see Fig. 5 for $\mathrm{Ra}=10^{7}$ and $\operatorname{Pr}=0.7$ ), which, according to Ref. [20], is already turbulent. The unstable TRS initial condition for $\Gamma=$ 0.63 falls back to the DRS, whereas for initial DRS condition at $\Gamma=0.64$ the flow remains in this DRS (with lower $\mathrm{Nu}$ ) for longer than $3000 t_{E}$. It remains to be seen whether this explicit dependence on initial conditions can also be found at larger $\mathrm{Ra}$.

We finally address the question as to why $\mathrm{Nu}$ is much more sensitive to the flow organization at small Pr as compared to large $\mathrm{Pr}-$ see again Fig. 1. The first reason for this finding is the organization of the thermal BL and kinetic BL: For large $\mathrm{Pr}$ the thermal BL is nested in the kinetic one. Thus modifications of the bulk flow are buffered by the kinetic BL and hardly lead to a different thermal BL thickness $\lambda_{\theta}$ and therefore to hardly any change in $\mathrm{Nu} \approx L /\left(2 \lambda_{\theta}\right)$. In contrast, for low Pr the thermal BL is thicker than the kinetic one and

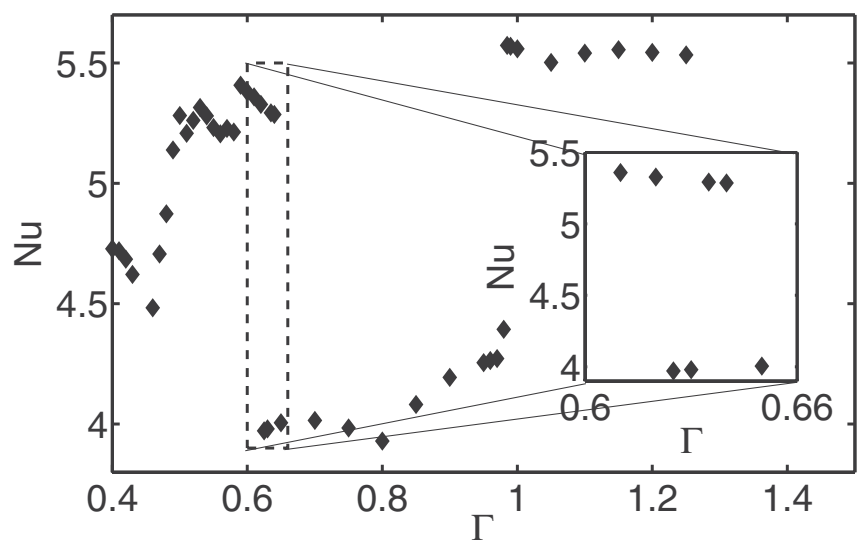

FIG. 5. $\mathrm{Nu}(\Gamma)$ for $\mathrm{Ra}=10^{7}$ and $\mathrm{Pr}=0.7$. For $\Gamma \approx 0.64$ the Nusselt number depends on the initial conditions - see the text for details.

is therefore fully exposed to bulk flow modifications, leading to a much stronger dependence of $\mathrm{Nu} \approx L /\left(2 \lambda_{\theta}\right)$ on the flow organization. The second reason for the larger sensitivity of $\mathrm{Nu}$ at smaller $\mathrm{Pr}$ is the larger thermal diffusivity, leading to a thermal smoothening of the plumes. As discussed above, this results in sharper transitions between different states. Our interpretation is supported by Fig. 6, in which we plot $\mathrm{Nu}(\Gamma)$ versus a normalized $\operatorname{Re}_{z}(\Gamma)$, where $\operatorname{Re}_{z}=u_{z, \mathrm{rms}} L / \nu$, which represents the bulk flow. For $\operatorname{Pr}=4.3$ there is hardly any dependence of $\mathrm{Nu}$ on $\mathrm{Re}_{z} /\left\langle\mathrm{Re}_{z}\right\rangle$, whereas for $\mathrm{Pr}=0.7$ this dependence is major. Here $\left\langle\operatorname{Re}_{z}\right\rangle$ is the average Reynolds number of the shown $\Gamma$ range $0.4 \leqslant \Gamma \leqslant 1.25$.

In conclusion, we could clearly identify different turbulent states, corresponding to different roll structures. It is remarkable that these features, which have been associated with lower Reynolds and Rayleigh number flow in the regime of spatial-temporal chaos with much less dynamical degrees of freedom, survive for such high $\mathrm{Ra}$. It seems that the turbulent states "live" on low-dimensional structures. The different turbulent states are associated with different overall heat transfers. Transitions between the states-either dynamically

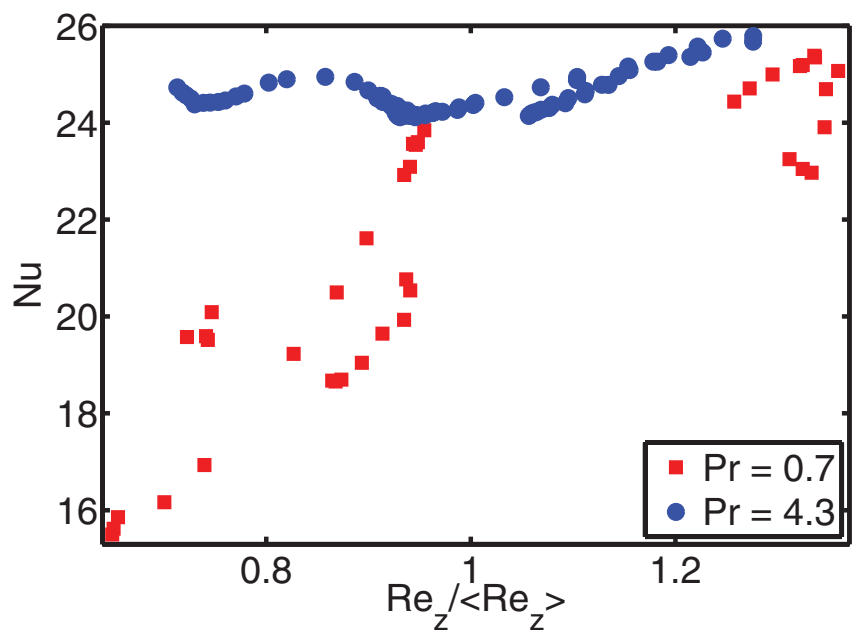

FIG. 6. (Color online) $\mathrm{Nu}(\Gamma)$ vs $\operatorname{Re}_{z}(\Gamma) /\left\langle\operatorname{Re}_{z}\right\rangle$ for $\operatorname{Pr}=0.7$ (red squares) and $\operatorname{Pr}=4.3$ (blue bullets) at $\mathrm{Ra}=10^{8}$. 
or as function of the control parameter $\Gamma$-thus imply jumps and sharp transitions in $\mathrm{Nu}(\Gamma)$. Based on the flow organization, we understand why these variations are larger for lower Pr and why the overall flow dynamics is richer at larger Pr, for which the plumes can keep their thermal identity for a longer time. The next step will be to push the present results to much higher $\mathrm{Ra}$ in order to see whether these features survive. Based on our analysis for $\mathrm{Ra}=10^{8}$ and $10^{9}$, we presume that this could be the case as the stability of the states and the variation in $\mathrm{Nu}(\Gamma)$ is higher for $\mathrm{Ra}=10^{9}$ compared to $\mathrm{Ra}=10^{8}$ at $\mathrm{Pr}=4.3$. Additionally, large-scale roll states have been distinguished up to $\mathrm{Ra}=10^{10}$ [24] and can probably be found for even higher Ra.

We thank K. Sugiyama for writing the first version of the numerical code. The work was supported by the Foundation for Fundamental Research on Matter (FOM) and the National Computing Facilities (NCF), both sponsored by NWO.
[1] A. N. Kolmogorov, Dokl. Akad. Nauk SSSR 30, 9 (1941) [reprinted in Proc. R. Soc. London A 434, 9 (1991)].

[2] P. P. Cortet, A. Chiffaudel, F. Daviaud, and B. Dubrulle, Phys. Rev. Lett. 105, 214501 (2010); R. Monchaux, F. Ravelet, B. Dubrulle, A. Chiffaudel, and F. Daviaud, ibid. 96, 124502 (2006); A. de la Torre and J. Burguete, ibid. 99, 054101 (2007).

[3] F. Ravelet et al., Phys. Rev. Lett. 101, 074502 (2008); R. Monchaux et al., Phys. Fluids 21, 035108 (2009); M. Berhanu et al., Eur. Phys. J. B 77, 459 (2010).

[4] R. J. A. M. Stevens, J. Q. Zhong, H. J. H. Clercx, G. Ahlers, and D. Lohse, Phys. Rev. Lett. 103, 024503 (2009); S. Weiss et al., ibid. 105, 224501 (2010).

[5] D. S. Zimmerman, A. A. Triana, and D. P. Lathrop, Phys. Fluids23, 065104 (2011); P. E. Roche, B. Castaing, B. Chabaud, and B. Hebral, Europhys. Lett. 58, 693 (2002); F. Chilla, M. Rastello, S. Chaumat, and B. Castaign, Euro. Phys. J. B 40, 223 (2004); C. Sun, H.-D. Xi, and K.-Q. Xia, Phys. Rev. Lett. 95, 074502 (2005); H.-D. Xi and K.-Q. Xia, Phys. Fluids 20, 055104 (2008); S. Weiss and G. Ahlers, J. Fluid Mech. 676, 5 (2011).

[6] G. Ahlers, D. Funfschilling, and E. Bodenschatz, New J. Phys. 13, 049401 (2011).

[7] S. Grossmann and D. Lohse, Phys. Fluids 23, 045108 (2011).

[8] P. E. Roche, G. Gauthier, R. Kaiser, and J. Salort, New J. Phys. 12, 085014 (2010).

[9] E. Bodenschatz, W. Pesch, and G. Ahlers, Annu. Rev. Fluid Mech. 32, 709 (2000).

[10] G. Ahlers, S. Grossmann, and D. Lohse, Rev. Mod. Phys. 81, 503 (2009).

[11] C. Sun, H. D. Xi, and K.-Q. Xia, Phys. Rev. Lett. 95, 074502 (2005); H. D. Xi and K.-Q. Xia, Phys. Fluids 20, 055104 (2008).

[12] S. Grossmann and D. Lohse, J. Fluid. Mech. 486, 105 (2003).

[13] E. S. C. Ching and W. S. Tam, J. Turbulence 7, 1 (2006).
[14] J. Bailon-Cuba, M. S. Emran, and J. Schumacher, J. Fluid Mech. 655, 152 (2010).

[15] D. Funfschilling, E. Brown, A. Nikolaenko, and G. Ahlers, J. Fluid Mech. 536, 145 (2005).

[16] A. Nikolaenko, E. Brown, D. Funfschilling, and G. Ahlers, J. Fluid Mech. 523, 251 (2005).

[17] C. Sun, L.-Y. Ren, H. Song, and K.-Q. Xia, J. Fluid Mech. 542, 165 (2005).

[18] E. E. DeLuca, J. Werne, R. Rosner, and F. Cattaneo, Phys. Rev. Lett. 64, 2370 (1990); J. Werne, E. E. DeLuca, R. Rosner, and F. Cattaneo, ibid. 67, 3519 (1991); J. Werne, Phys. Rev. E 48, 1020 (1993).

[19] J. Schmalzl, M. Breuer, and U. Hansen, Geophys. Astrophys. Fluid Dyn. 96, 381 (2002); J. Schmalzl, M. Breuer, S. Wessling, and U. Hansen, Europhys. Lett. 67, 390 (2004).

[20] K. Sugiyama, E. Calzavarini, S. Grossmann, and D. Lohse, Europhys. Lett. 80, 34002 (2007).

[21] G. Ahlers et al., Phys. Rev. E 77, 046302 (2008).

[22] K. Sugiyama, E. Calzavarini, S. Grossmann, and D. Lohse, J. Fluid Mech. 637, 105 (2009).

[23] H. Johnston and C. R. Doering, Phys. Rev. Lett. 102, 064501 (2009).

[24] K. Sugiyama et al., Phys. Rev. Lett. 105, 034503 (2010).

[25] Q. Zhou et al., J. Fluid Mech. 664, 297 (2010); M. Chandra and M. K. Verma, Phys. Rev. E 83, 067303 (2011).

[26] R. J. A. M. Stevens, R. Verzicco, and D. Lohse, J. Fluid Mech. 643, 495 (2010).

[27] See Supplemental Material at http://link.aps.org/supplemental/ 10.1103/PhysRevE.84.045303 for flow field movies.

[28] R. Halír and J. Flusser, Proc. Sixth International Conference Computer Graphics and Visualization 1, 125 (1998).

[29] S. Weiss and G. Ahlers, J. Fluid. Mech. 676, 5 (2010). 